\title{
Dynamique centrifuge du maraîchage périurbain de Lomé (Togo) en réponse à la pression foncière
}

\author{
Madjouma Kanda ${ }^{1, *}$, Hèou Maléki Badjana ${ }^{1}$, Fousseni Folega ${ }^{1}$, Sêmihinva Akpavi ${ }^{1}$, Kpérkouma Wala ${ }^{1}$, \\ Jacques Imbernon $^{2}$ et Koffi Akpagana ${ }^{1}$ \\ ${ }^{1}$ Laboratoire de Botanique et Écologie végétale, Faculté des Sciences, Université de Lomé, BP 1515, Lomé, Togo \\ 2 CIRAD-Département Environnement et Sociétés, UMR TETIS, TA C-91/F, Campus International de Bazillarguet, 34398 Montpellier, \\ France
}

\begin{abstract}
Résumé - Cette étude, menée à l'est de la ville de Lomé (capitale et plus grande ville du Togo), met en évidence la dynamique spatiale des cultures maraîchères en réponse à la pression foncière. Des images satellitaires TerraMetrics des archives Google Earth des années 2002, 2008 et 2014 ont été retenues pour l'analyse de l'évolution des périmètres maraîchers. Une enquête de terrain fondée sur des questionnaires semistructurés a été menée pour identifier les stratégies des maraîchers. Les coordonnées géographiques des points d'observation ont été relevées à l'aide d'un capteur GPS. Les superficies des périmètres maraîchers ont été évaluées grâce au logiciel QGIS couplé avec le tableur Excel. L'évolution spatiale et descriptive des superficies maraîchères depuis 2002 a été ainsi connue. Il a été également possible de comprendre la dynamique spatiotemporelle des superficies ainsi que d'évaluer les stratégies développées par les maraîchers pour faire face à la pénurie foncière. Ces périmètres jouissent d'un statut foncier précaire ; leur superficie, grignotée par l'urbanisation, a diminué en moyenne de 31 hectares par an entre 2002 et 2014, chutant de 530 à 160 ha.
\end{abstract}

Mots clés : foncier / maraîchage / agriculture péri-urbaine / Lomé

\begin{abstract}
Peri-urban market gardening centrifugal dynamics in Lomé (Togo) in response to land pressure. This study, conducted in the eastern part of the city of Lomé (capital and largest city of Togo), highlights the spatial dynamics of market gardening under land pressure. TerraMetrics satellite images from Google Earth archives for 2002, 2008 and 2014 were selected for the market gardening evolution analysis. A field survey based on semi-structured questionnaires was conducted to identify the gardeners' strategies. The observation points that geographical coordinates were recorded using a GPS sensor. Garden areas were evaluated using the QGIS software coupled with the Excel spreadsheet. The spatial and descriptive evolution of the market gardening areas since 2002 has thus been known. It was also possible to understand the spatial and temporal dynamics of these areas and to evaluate the strategies developed by the gardeners to cope with the land shortage. Market gardening land status is precarious; in average, surveyed gardeners lost 31 ha per year between 2002 and 2014, the total market gardening surface area falling from 530 to 160 ha.
\end{abstract}

Keywords: land issues / market gardening / peri-urban agriculture / Lomé

\section{Introduction}

La population urbaine des pays d'Afrique subsaharienne sera de 300 millions en 2020, avec un taux de croissance de $3,7 \%$ par an (Véron, 2007). Pour répondre à ce défi, il conviendra de prendre en compte et d'améliorer la production agricole en ville. Étant donnée la faiblesse des secteurs agricoles habituels, l'agriculture urbaine et périurbaine peut être un atout. La demande de plus en plus forte des villes en légumes frais et le manque d'emploi favorisent le

\footnotetext{
* Auteur correspondant : kmadjouma@gmail.com
}

développement de cette agriculture proche des consommateurs (Delamarche, 2007 ; Dieye, 2006). Elle permet d'assurer un approvisionnement régulier de produits marâichers de qualité en limitant le coût du transport.

Toutefois en milieu urbain, l'insécurité foncière liée au mode de faire valoir (statut précaire de location) et la raréfaction des terres disponibles (Bertrand et Roussier, 2004 ; Dauvergne, 2012) restent à maîtriser. L'urbanisation, la légitimation de la place de l'agriculture dans le plan urbain ainsi que son intégration dans la gestion du paysage et de l'espace conditionnent la pérennité de cette agriculture (Boudjenouia et al., 2008 ; Fleury et Donadieu, 1997). En effet, l'étalement des villes engendre une compétition foncière 
qui ne laisse guère d'espaces pour les activités agricoles. Lomé, capitale du Togo, n'échappe pas à ce mouvement général d'urbanisation. Cette croissance urbaine se traduit par une forte demande en terres à bâtir et est largement alimentée par le développement des maisons individuelles (Guézéré, 2011). Dans ce contexte, que devient la production agricole à Lomé ? Quels sont ses enjeux par rapport au foncier ? Comment évoluent les superficies dans le temps et dans l'espace ?

Pour répondre à ces questions, cette étude propose de contribuer à la connaissance de la dynamique des périmètres maraîchers périurbains de Lomé.

\section{Méthodologie}

\subsection{Contexte géographique}

La zone d'étude (Fig. 1 et 2) est située sur le cordon littoral, entre $1^{\circ} 14^{\prime} 0$ " et $1^{\circ} 24^{\prime} 0$ " de longitude est, et $6^{\circ} 7$ ' $12^{\prime \prime}$ et $6^{\circ} 12^{\prime} 0$ " ' de latitude nord. Elle jouit d'un climat subéquatorial guinéen à régime bimodal ; la moyenne annuelle des précipitations est de $860 \mathrm{~mm} / \mathrm{an}$ et l'humidité relative de 80 à $90 \%$; l'évapotranspiration moyenne est de $1540 \mathrm{~mm} / \mathrm{an}$ et la température moyenne varie de 26 à $33^{\circ} \mathrm{C}$. Les sols, développés sur les sables littoraux, sont pauvres en matières organiques et en éléments minéraux ; ils sont perméables et leur réserve en eau est faible.

De 1981 à 2010, la population de la commune de Lomé et la partie urbaine de la Préfecture du Golfe est passée de 390000 à 1477660 habitants (DRSP, 2012). La commune de Lomé comptait à elle seule 840000 habitants en 2012 et est de loin, la plus grande cité du Togo ; le taux d'urbanisation est estimé à $55 \%$ (Janssens, 1998). Du fait de l'accès facilité à l'eau, cette zone est propice à la production maraîchère et approvisionne la ville de Lomé en légumes frais (Kanda et al., 2009).

\subsection{Collecte des données}

Les images satellitaires et les données d'enquête de terrain ont constitué la base de cette étude (Kêdowidé et al., 2010). Ces images sont de type TerraMetrics de résolution spatiale maximale de $15 \mathrm{~m}$ x $15 \mathrm{~m}$ extraites d'archives Google Earth. Trois années (2002, 2008 et 2014) ont été retenues en fonction de la disponibilité spatiale et temporelle des images mais aussi de leur qualité permettant l'identification aussi précise que possible des surfaces maraîchères. Le maraîchage se pratiquant en continu toute l'année, il n'y a pas de variation phénologique importante entre les images des différentes années retenues quel que soit le mois ou la saison. Étant donnée la résolution spatiale maximale des images et la taille minimale d'un site agricole $\left(302 \mathrm{~m}^{2}\right)$, les surfaces maraîchères pouvaient être identifiées clairement sans confusion avec d'autres types d'occupation du sol.

L'unité de sondage choisie est le site agricole. Le site est un espace de culture constitué d'une ou de plusieurs parcelles contiguës cultivées par un producteur.

Les données descriptives permettant de décrire l'activité et les données spatiales pour situer géographiquement les sites agricoles sur une carte et calculer leurs superficies ont été collectées. Des entretiens semi-directifs individuels et en « focus group » ont été réalisés auprès de 78 maraîchers échantillonnés de manière aléatoire le long d'un transect ouestest suivant le littoral. À ce jour il n'existe pas de données statistiques précises et à jour du nombre de maraîchers dans la ville de Lomé. Néanmoins, la taille de l'échantillon qui représente environ $20 \%$ des estimations des maraîchers de la ville de Lomé en 1987 (Schilter, 1991), est considérée comme représentative car les enquêtes ont concerné la majorité des maraîchers de la zone d'étude. Le questionnaire a porté essentiellement sur le statut de l'exploitation (localisation de la parcelle, superficie exploitée, mode de faire valoir, durée d'occupation, évolution des parcelles depuis 2000, etc.) ainsi que les stratégies paysannes de maintien de l'activité.

Les coordonnées géographiques et les superficies des sites agricoles ont été prises à l'aide d'un capteur GPS Garmin ${ }^{\mathrm{TM}}$ Oregon 550t dont la précision est inférieure à 10 mètres.

Pour chaque année, toutes les parcelles maraîchères ont été digitalisées directement dans Google Earth de manière exhaustive sous forme de polygones. La carte IGN de la Région Maritime au $1 / 200000^{\mathrm{e}}$ de 1980 et 2013 ainsi que des documents de la littérature existant sur le sujet ont été également consultés.

\subsection{Traitement des données}

Les données collectées au GPS ont été intégrées dans QGIS afin de visualiser l'emplacement des sites d'enquête sur un fond de carte. Les différents traitements ont permis d'avoir les situations spatiales et descriptives pour les trois années 2002, 2008 et 2014 et de déceler la dynamique spatiale.

À l'exception de l'image de 2014, celles de 2002 et 2008 possédaient des plages floues créant ainsi des données manquantes. Certaines données manquantes de 2002 ont été complétées par celles collectées directement sur le terrain en 2002 au cours de précédentes études (Kanda et al., 2009). D'autres ont été générées à partir d'images d'archives Google Earth de 2003 et 2004 suite à des enquêtes rétrospectives auprès des maraîchers installés depuis 2000 dans la zone. De la même manière, l'image de 2009 a servi à compléter les données manquantes de 2008.

Les données digitalisées dans Google Earth ont été sauvegardées sous format $\mathrm{kml}$ puis chargées dans le logiciel QGIS v.2.4.0 où elles sont converties en données vectorielles sous format .shp (ESRI Shapefile). Les superficies ont été calculées et leur précision évaluée en calculant pour les mêmes parcelles, les erreurs entre les superficies obtenues par digitalisation et celles mesurées directement sur le terrain à l'aide du GPS (Nguyen et al., 2004). L'intersection des différents fichiers vectoriels a été réalisée pour estimer et illustrer les superficies restées inchangées, perdues et ajoutées d'une année à l'autre. L'analyse de ces modifications a été effectuée entre 2002 et 2008, 2008 et 2014 et 2002 et 2014.

Les données d'enquête ont été dépouillées à l'aide du logiciel de traitement statistique SPSS (Statistical Package for The Social Science) v. 16.

\section{Résultats}

\subsection{Des superficies perdues pour le maraîchage du fait de l'urbanisation}

Les Figures 3A, B et C montrent respectivement la distribution des surfaces maraîchères en 2002, 2008 et 2014 dans les secteurs du Port autonome de Lomé, de la raffinerie de pétrole et de l'Hôtel Novela Star. 


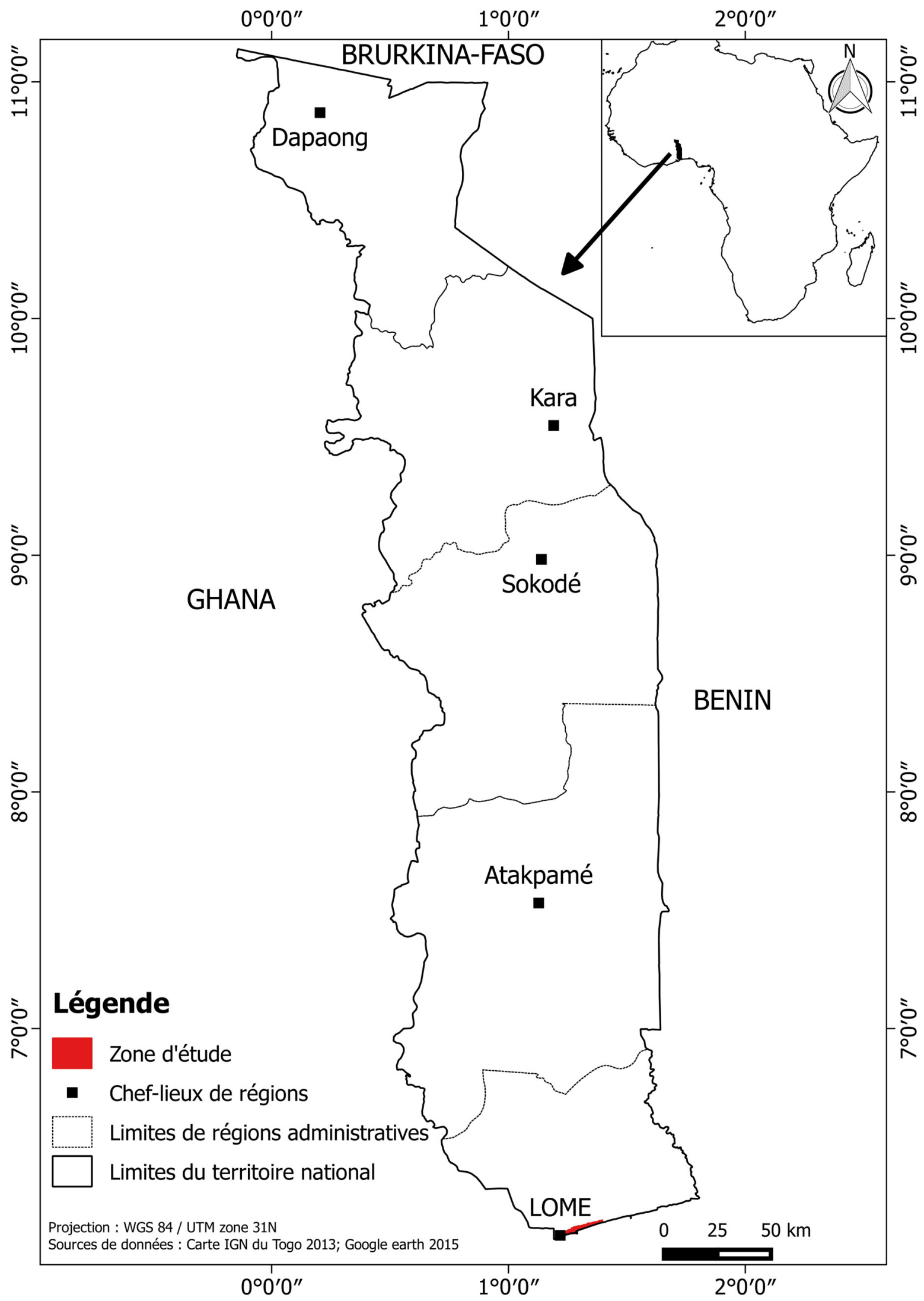

Fig. 1. Présentation du Togo (Afrique de l'Ouest) montrant la zone d'étude (en rouge).

Fig. 1. Presentation of Togo (West Africa) showing the study zone (in red). 


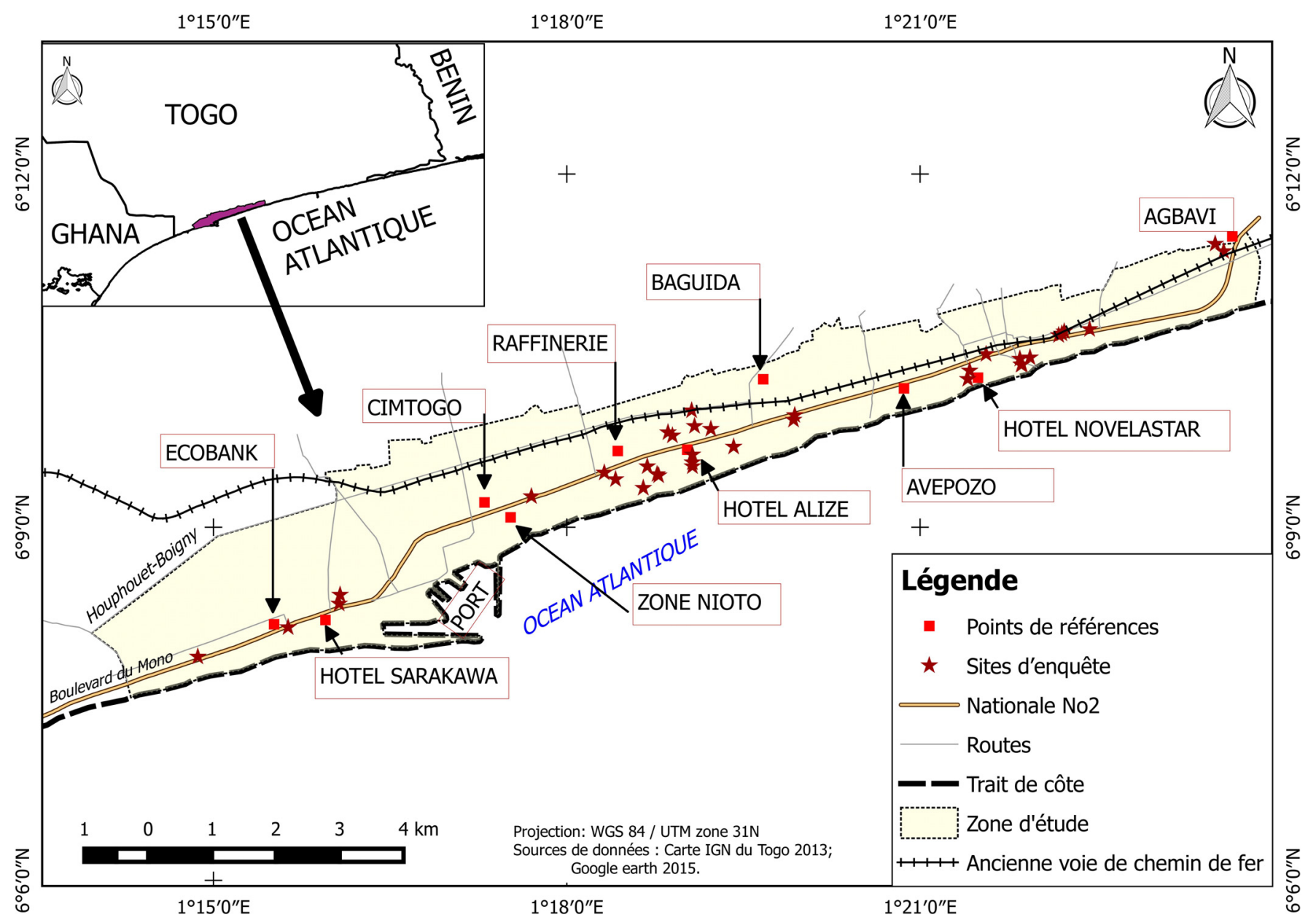

Fig. 2. Présentation de la zone d'étude montrant les sites d'enquête (étoiles).

Fig. 2. Study zone presentation with the survey sites (stars).

En 2002 (Fig. 3A), de grandes étendues de surfaces maraîchères dans les secteurs du Port autonome de Lomé et de la raffinerie de pétrole sont observées. Ceci s'explique par une plus grande disponibilité de terres dans ces secteurs permettant l'installation d'agriculteurs. La superficie totale des périmètres maraîchers a été estimée à $532 \pm 16$ hectares.

En 2008 (Fig. 3B), alors que les secteurs de la raffinerie de pétrole et de l'Hôtel Novela Star se conservent plus ou moins bien, on observe un émiettement de celui du Port autonome de Lomé réduisant les surfaces maraîchères à $383 \pm 11$ hectares. Cette réduction de surfaces s'explique d'une part, par la réhabilitation et la modernisation des capacités manutentionnaires du Port autonome de Lomé et d'autre part, par l'érection d'immeubles administratifs ; les maraîchers ont ainsi été contraints de se déplacer.

En 2014 (Fig. 3C), l'émiettement de tous les secteurs se poursuit. L'aménagement du secteur du Port autonome de Lomé s'est accentué avec l'achèvement des travaux de réhabilitation et de modernisation. L'étendue de la zone portuaire a été multipliée par un facteur proche de 0,6 entraînant la cessation de toute activité agricole dans ses environs immédiats et l'éviction des maraîchers. Dans le même temps, les secteurs de la raffinerie de pétrole et de l'Hôtel
Novela Star connaissent une forte urbanisation, réduisant de fait les activités agricoles. La superficie des périmètres maraîchers a été estimée à $163 \pm 5$ hectares.

De 2002 à 2014, les superficies maraîchères ont régressé (Fig. 4) de 31 ha/an ; ce qui pourrait compromettre à terme le maraîchage dans la zone étudiée.

La disparition des parcelles en certains endroits est compensée par la mise en place ailleurs, de nouvelles parcelles. Ainsi, sur 532 ha de surfaces maraîchères de 2002, 212 ha (soit $40 \%$ ) ont été perdus en 2008 et $60 \%$ sont restés inchangés, tandis que 63 ha ont été ajoutés (Tab.1, Fig. 5). Également, sur 383 ha en 2008,269 ha, soit $70 \%$ ont été perdue, $30 \%$ sont restés inchangés alors que seulement 49 ha ont été ajoutés. La comparaison entre les situations de 2002 et 2014 montre que sur les 532 ha de 2002, 416 ha, soit $78 \%$ ont été perdus tandis que 47 ha ont été ajoutés. Par ailleurs, les surfaces maraîchères inchangées en 2002, 2008 et 2014 sont estimées à 104 ha, soit $20 \%$ de celles de 2002 ; celles-ci sont surtout localisées vers raffinerie de pétrole et l'Hôtel Novela Star. Les surfaces maraîchères abandonnées définitivement se répartissent sur toute la zone d'étude. Toutefois, le secteur du Port autonome de Lomé semble plus impacté. 

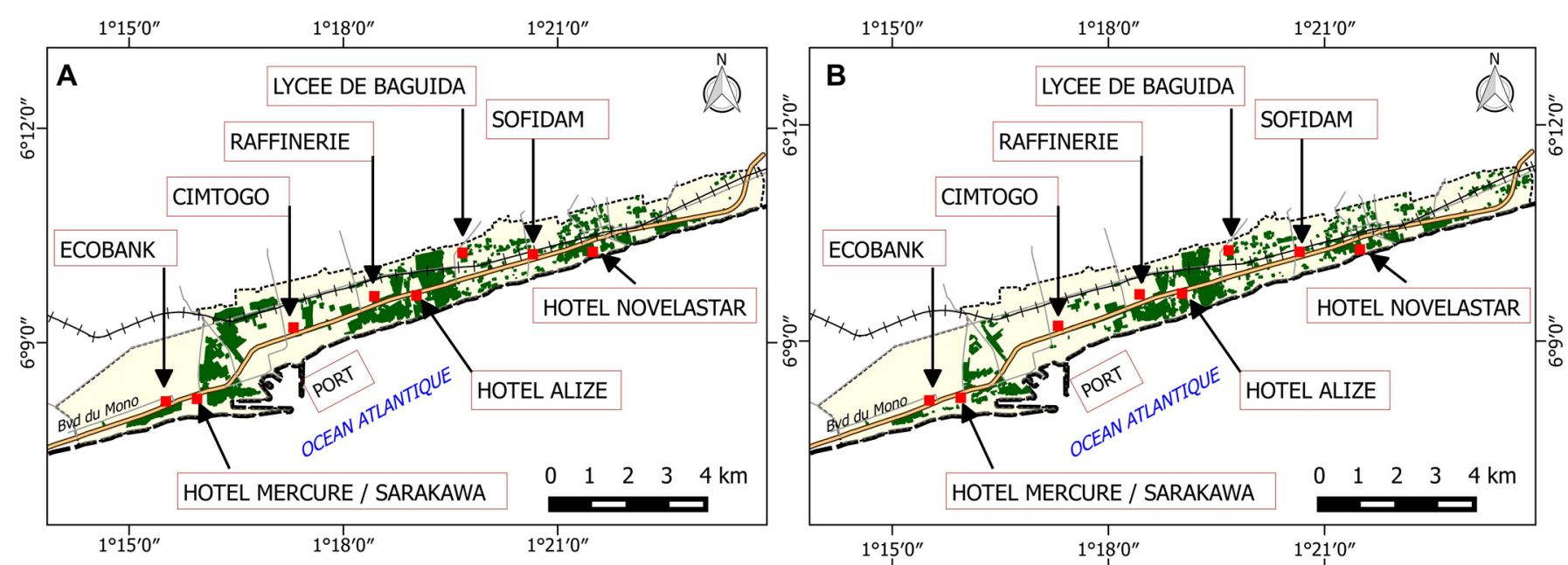

\section{Légende}

- Points de références Routes

Nationale $\mathrm{N}^{\circ} 2$

:....... Zone d'étude

Surfaces maraîchères

+1 Ancienne voie de chemin de fer

- Trait de côte

Projection : WGS 84 / UTM zone 31N

Sources de données : Cartes IGN du Togo 2013; Google earth 2002, 2003. 2004. 2008. 2009. 2014 et 2015.

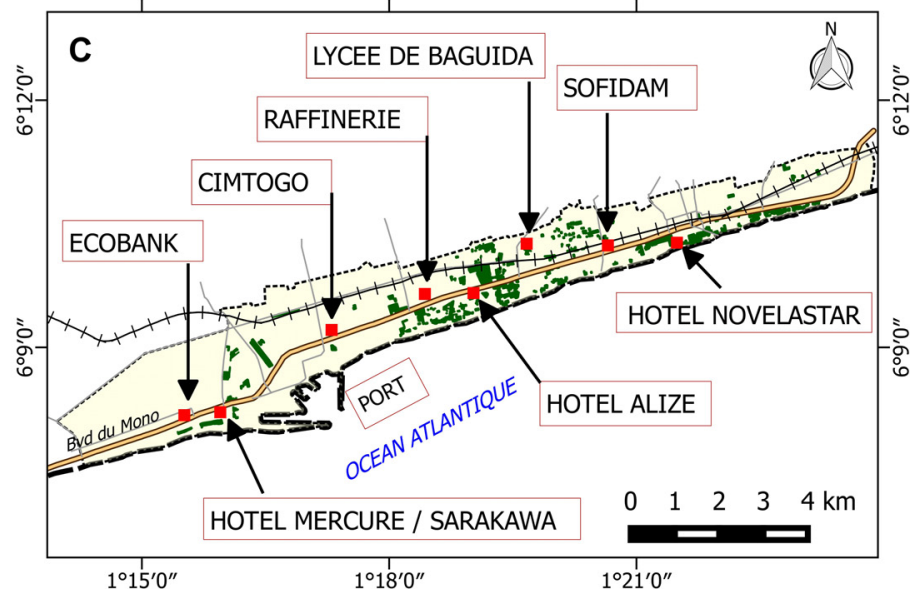

Fig. 3. Superficies occupées par l'agriculture maraîchère en 2002 (A), 2008 (B) et 2014 (C).

Fig. 3. Areas under market gardening in 2002 (A), 2008 (B) and 2014 (C).

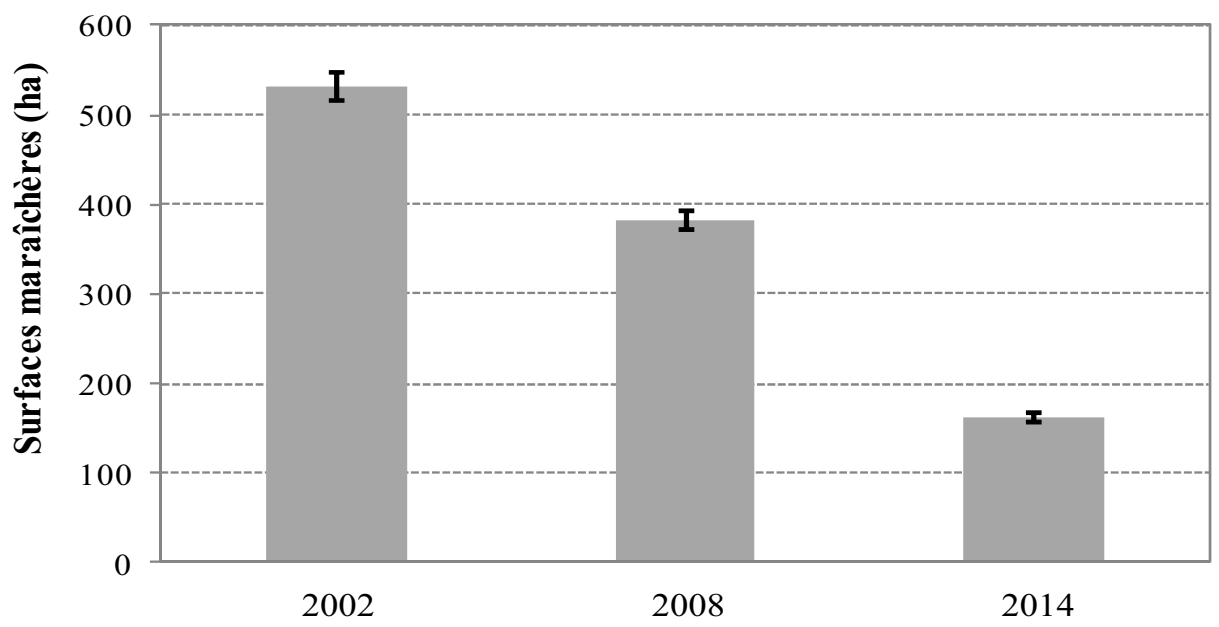

Années

Fig. 4. Évolution des surfaces maraîchères entre 2002 et 2014.

Fig. 4. Evolution of market gardening areas from 2002 to 2014. 
Tableau 1. Évolution des superficies maraîchères.

Table. Market gardens' acreages evolution.

Surfaces en hectare (pourcentage de la superficie de l'année de départ)

Surfaces maraîchères ajoutées

Surfaces maraîchères perdues

Surfaces maraîchères inchangées

$\begin{array}{lll}2002-2008 & 63 & 212(40 \%) \\ 2008-2014 & 49 & 269(70 \%) \\ 2002-2014 & 47 & 416(78 \%)\end{array}$

$320(60 \%)$

$114(30 \%)$

$116(22 \%)$

Surfaces maraîchères de 2002 restées inchangées en 2008 et 2014

$104(20 \%)$

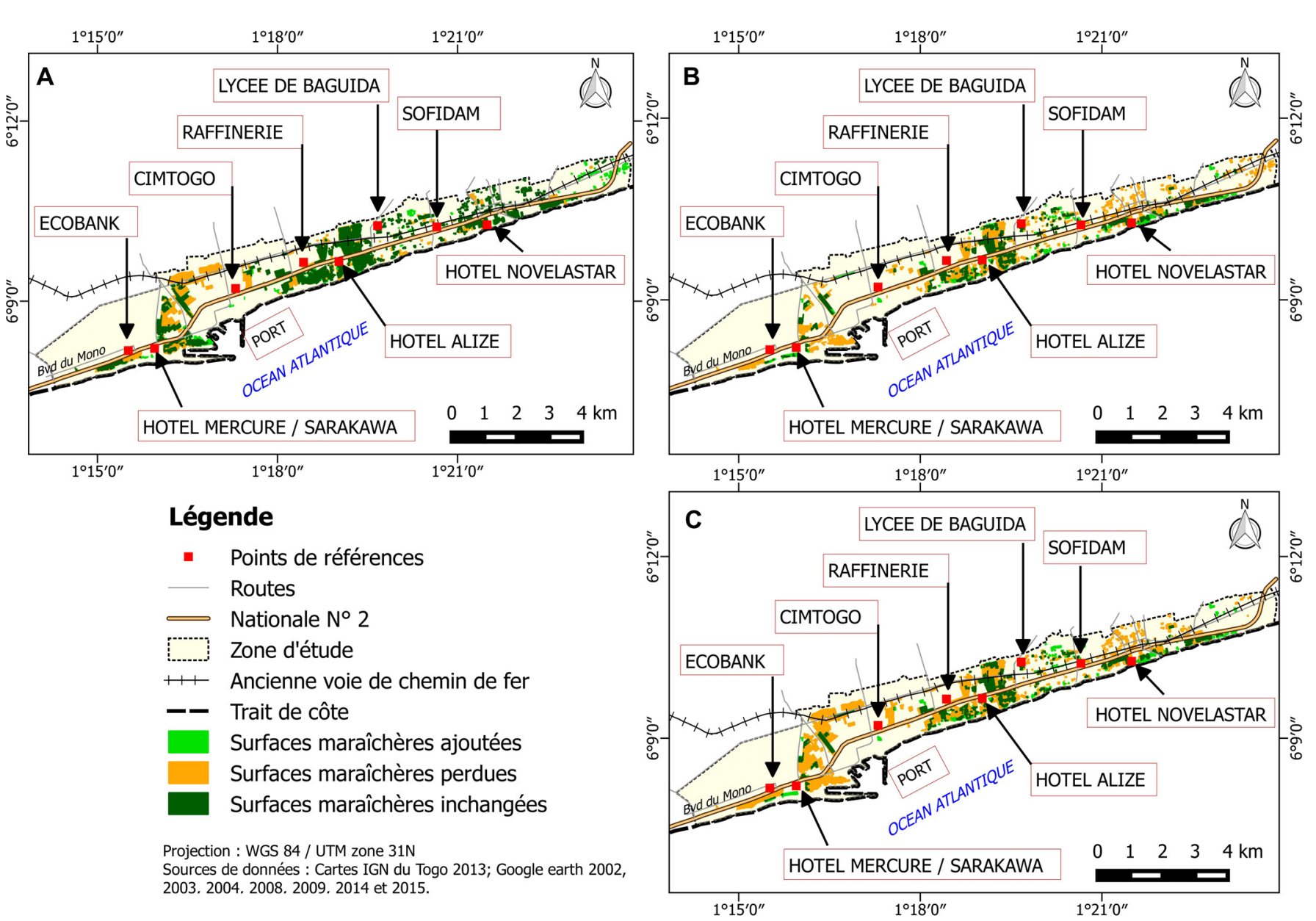

Fig. 5. Changements dans les superficies maraîchères entre 2002-2008 (A), 2008-2014 (B) et 2002-2014 (C).

Fig. 5. Changes in vegetable acreages between 2002-2008 (A), 2008-2014 (B) and 2002-2014 (C).

\subsection{Des parcelles de petite taille et à statut foncier précaire}

Les superficies maraîchères varient de moins d'un hectare à trois hectares. Celles de moins d'un hectare sont les plus représentées $(71 \%)$.

Trois types de propriétés foncières ont été identifiés (Fig. 6) : les terres appartenant à des privés (personnes physiques et sociétés) (53\%), les réserves administratives $(38 \%)$ et les terres familiales $(9 \%)$.

Les parcelles s'obtiennent par le don temporaire (56\%), la location (43\%) et l'héritage $(1 \%)$. Le don temporaire concerne les domaines administratifs (27\%) (Fig. 7). Il s'agit de terrains urbains appartenant à l'État qui les attribue à des entreprises de la zone franche. Il prend également en compte des parcelles appartenant à des privés $(17 \%)$. La location est annuelle moyennant le versement d'une somme après entente avec le propriétaire. Elle concerne les parcelles appartenant à des privés $(25 \%)$.

Les parcelles maraîchères jouissent ainsi d'un statut foncier précaire et leur occupation reste temporaire. Les maraîchers ont juste le droit de pratiquer leur activité jusqu'à ce que la parcelle soit aménagée par l'État ou le privé. Aussi, se déplacent-ils pour continuer à exercer leur activité.

\subsection{Des maraîchers contraints au déplacement et sans alternatives}

La récupération des terres pour la construction d'infrastructures $(79 \%)$ est le principal motif de déplacement des 


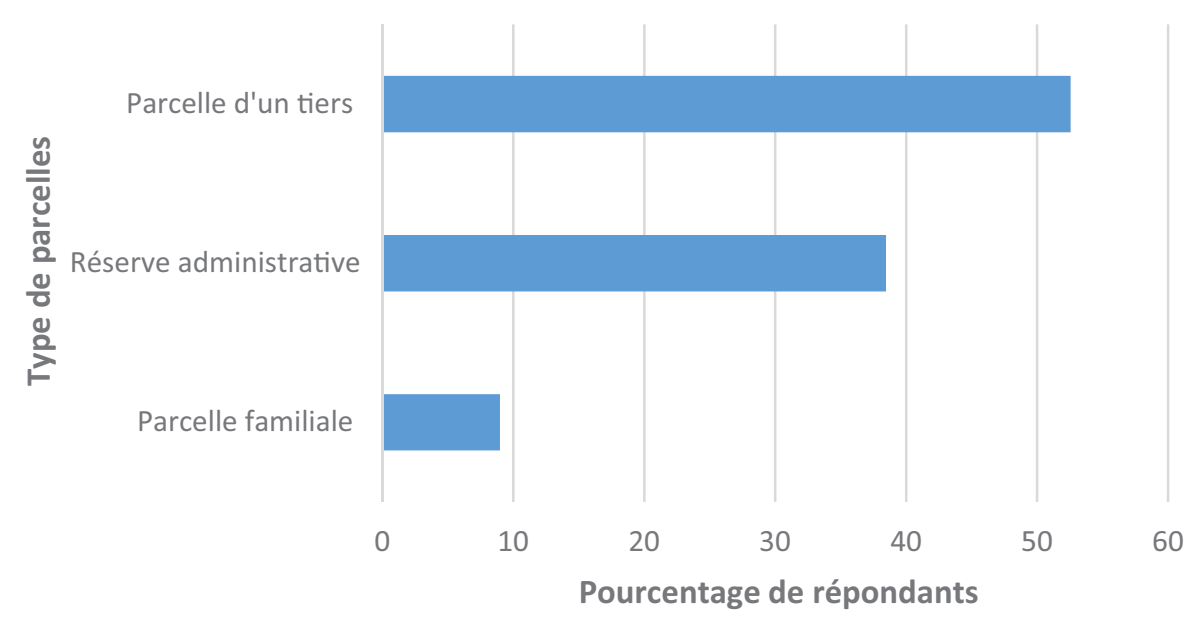

Fig. 6. Répartition des propriétés foncières.

Fig. 6. Distribution of landholdings.

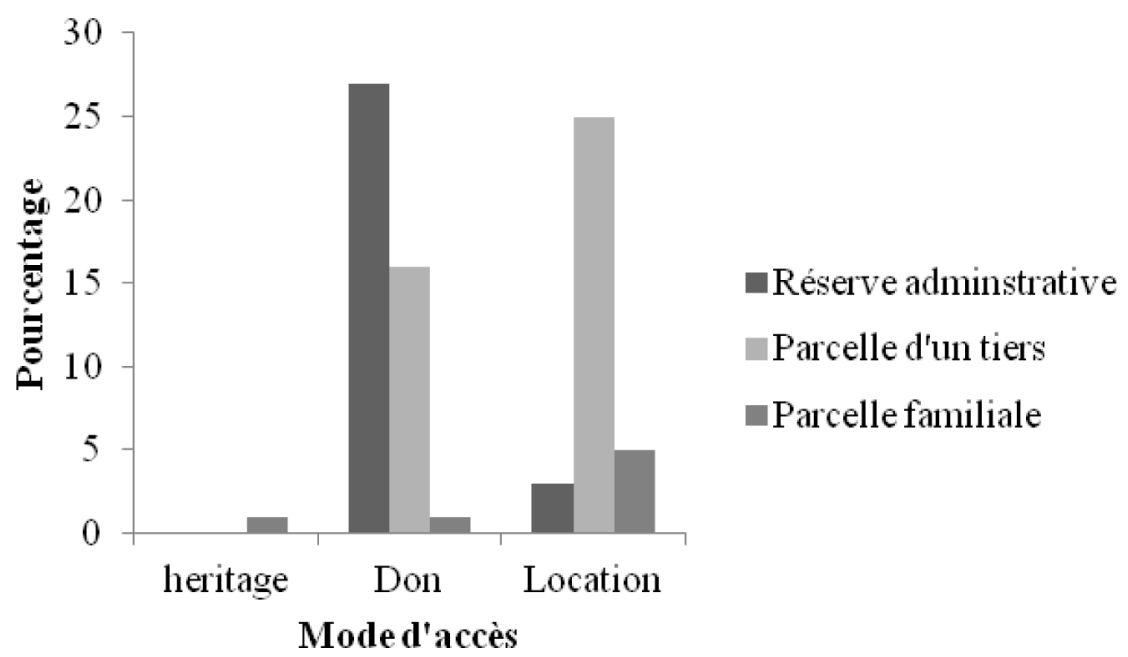

Fig. 7. Accès au foncier en fonction de la propriété foncière.

Fig. 7. Access to land based on land ownership.

maraîchers (Tab. 2). Ces déplacements se font de l'ouest vers l'est (Fig. 8). Les taux de déplacement les plus élevés sont localisés dans les secteurs du Port autonome de Lomé et de la raffinerie de pétrole.

Soixante pour cent des interviewés n'ont aucune alternative en cas de perte de leur périmètre (Fig. 9). Trente-trois pour cent déclarent qu'ils vont rechercher des terres ailleurs sans toutefois préciser où ils comptent trouver ces terres. Cela dénote leur désarroi. La superficie et la population de la ville de Lomé ne cessent de croître ; cette croissance nonmaîtrisée ne donne aucune alternative aux maraîchers en recherche de terres cultivables.

\section{Discussion}

Dans l'optique de contribuer à la connaissance de la dynamique des surfaces maraîchères et la durabilité du maraîchage dans la ville de Lomé, une analyse de l'imagerie satellitaire combinée à des investigations de terrain a été menée.
L'analyse a permis d'estimer les surfaces maraîchères pour chaque année retenue et d'étudier leur évolution ainsi que les facteurs qui la provoquent.

Les statistiques de l'évolution des surfaces maraîchères constituent une valeur ajoutée pour la thématique de l'agriculture périurbaine à Lomé sur laquelle des données de ce genre n'existent pas. Les incertitudes relativement grandes des superficies obtenues peuvent être liées à :

- la petite taille des surfaces maraîchères pour lesquelles les mesures avec le GPS auraient des erreurs élevées. En effet, plus la parcelle est petite, plus grande est l'erreur relative de la mesure avec le GPS (Nguyen et al., 2004) ;

- la prise en compte lors de la digitalisation, de petites surfaces qui ne sont pas concernées par les cultures mais qui s'intercalent entre les périmètres maraîchers et les clôtures lorsque les champs sont clôturés alors que la digitalisation a été faite parfois sur les clôtures ;

- des distorsions géométriques causées par la variation de l'altitude au sol, la courbure de la terre, etc. (Billen et Cornélis, 2000). 
Tableau 2. Les raisons qui poussent les paysans au déplacement.

Table 2. Reasons why farmers move.

\begin{tabular}{|c|c|c|c|c|c|c|c|}
\hline $\begin{array}{l}\text { Site précédemment } \\
\text { occupé }\end{array}$ & $\begin{array}{l}\text { Terrain récupéré } \\
\text { pour construction } \\
\text { d'infrastructure }\end{array}$ & $\begin{array}{l}\text { Besoin } \\
\text { de terres }\end{array}$ & $\begin{array}{c}\text { Terrain récupéré } \\
\text { par le } \\
\text { propriétaire }\end{array}$ & $\begin{array}{l}\text { Pour se mettre } \\
\text { à son } \\
\text { propre compte }\end{array}$ & $\begin{array}{c}\text { Destruction } \\
\text { des cultures } \\
\text { par les bêtes }\end{array}$ & $\begin{array}{c}\text { Coupure } \\
\text { de courant }\end{array}$ & $\begin{array}{c}\text { Terrain } \\
\text { insuffisant }\end{array}$ \\
\hline Port & 6 & 0 & 0 & 0 & 0 & 0 & 0 \\
\hline Akodessewa & 4 & 0 & 1 & 0 & 0 & 0 & 0 \\
\hline Raffinerie & 4 & 1 & 0 & 1 & 0 & 0 & 0 \\
\hline Ablogame & 3 & 0 & 0 & 0 & 0 & 0 & 0 \\
\hline Cimtogo & 3 & 0 & 0 & 0 & 1 & 0 & 0 \\
\hline Avepozo & 2 & 0 & 0 & 0 & 0 & 0 & 0 \\
\hline Gbodjome & 1 & 1 & 0 & 0 & 0 & 1 & 0 \\
\hline $\begin{array}{l}\text { Hôtel Mercure } \\
\text { Sarakawa }\end{array}$ & 1 & 0 & 0 & 0 & 0 & 0 & 0 \\
\hline Alogavi & 0 & 0 & 0 & 0 & 0 & 0 & 1 \\
\hline Total & 41 & 5 & 2 & 1 & 1 & 1 & 1 \\
\hline Pourcentage & 79 & 10 & 3 & 2 & 2 & 2 & 2 \\
\hline
\end{tabular}

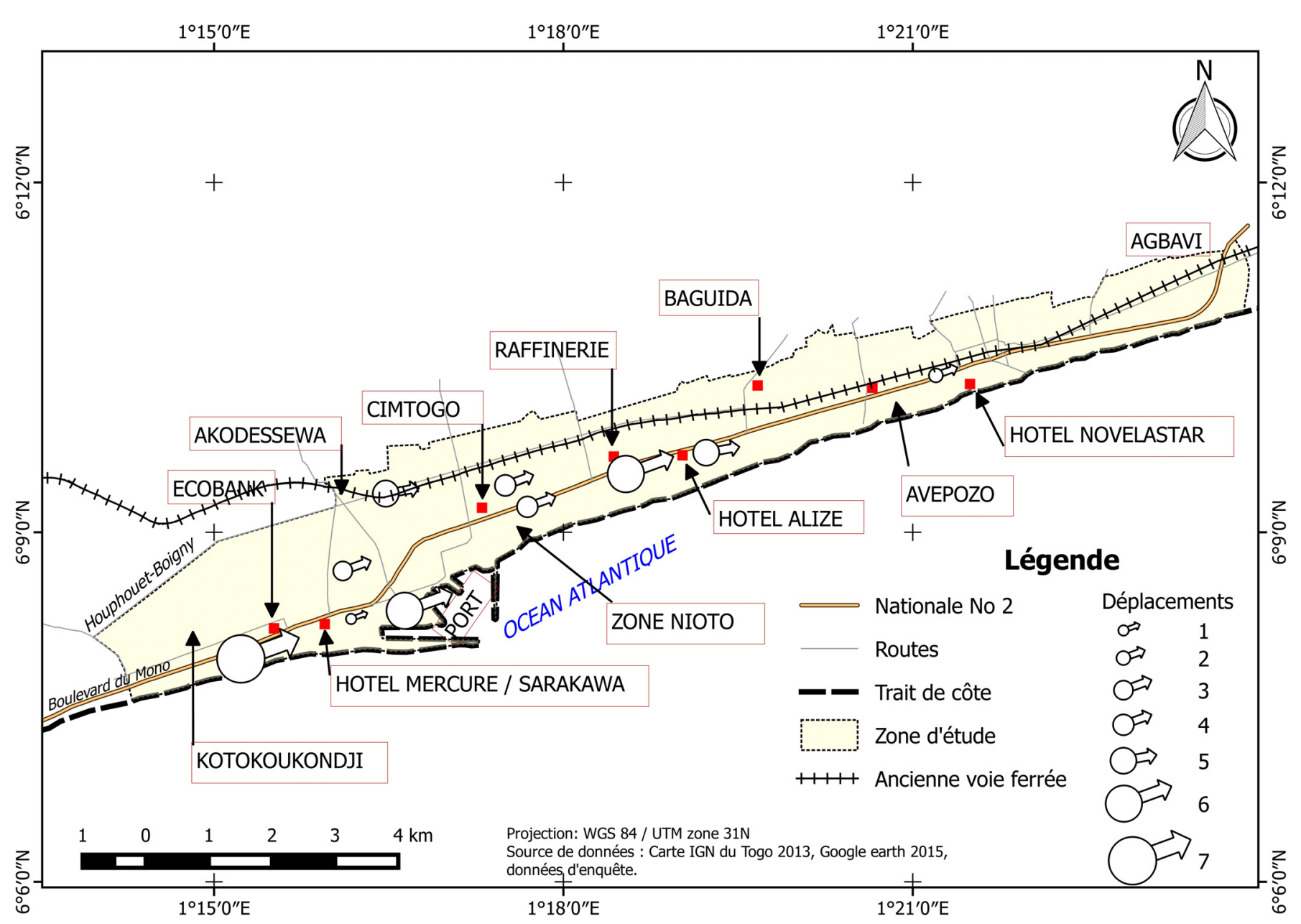

Fig. 8. Sens des déplacements des agriculteurs en fonction de la dynamique foncière.

Fig. 8. Farmers movements according to land dynamics. 


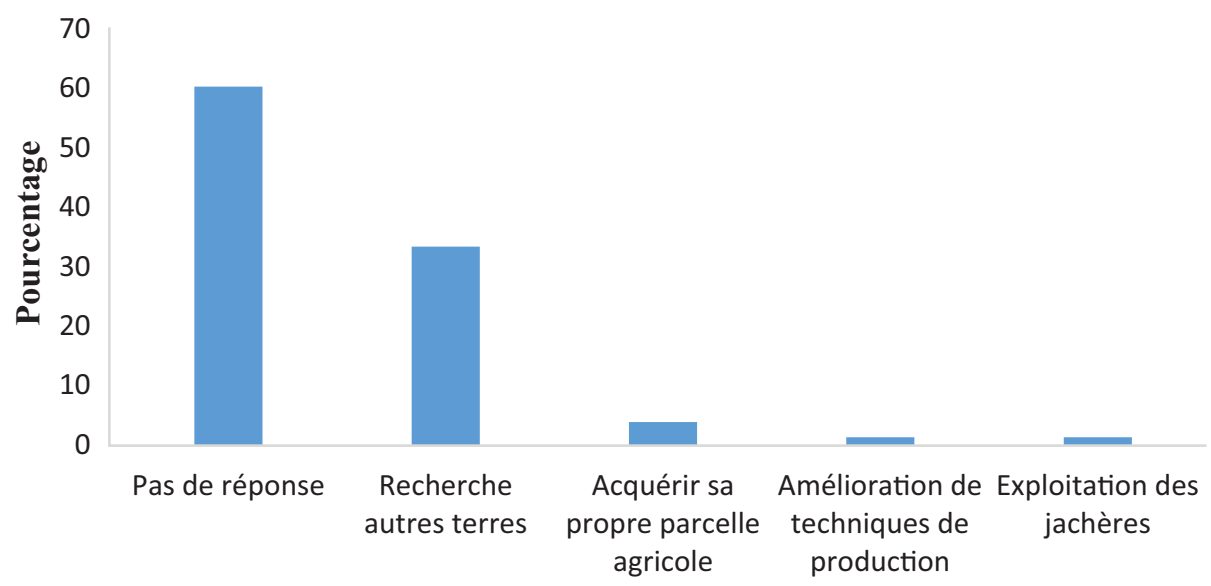

Alternatives

Fig. 9. Les alternatives développées par les paysans face à la pénurie foncière.

Fig. 9. Alternatives developed by farmers facing land scarcity.

La cartographie de l'occupation du sol (Cissé et al., 1999) est un intéressant outil d'évaluation des dynamiques observées sur l'activité agricole. L'étude a permis de mesurer la régression des surfaces maraîchères cultivables entre 2002 et 2014 au profit des constructions. Bien que l'urbanisation se poursuive, l'agriculture maraîchère se développe tant bien que mal sur des terres récupérées entre les maisons et les infrastructures industrielles en cours d'installation ou déjà installées. Cette situation n'est pas singulière à Lomé. L'impact négatif de la demande en terres cultivables sur le maraîchage a été mis en évidence à Abidjan (Kouakou et al., 2010) et à Ouagadougou (Kêdowidé et al., 2010). Il en est de même dans la plupart des villes africaines (Bélières et al., 2013 ; Boudemagh, 2010 ; Burnod et Tonneau, 2013 ; Valette et al., 2013). Dans ces villes tout comme à Lomé, les espaces jadis considérés comme propices à l'agriculture à leur abord immédiat, ont été, à un moment, l'objet de multiples convoitises et, dans les rapports de force qui s'instaurent, l'agriculture est rarement en position dominante. Dans ces conditions, la surface agricole utile ne peut connaître qu'une chute drastique, contribuant à la réduction des surfaces exploitées par les maraîchers. En effet dans notre étude, les superficies comprises entre 0,009 et 1 ha sont les plus représentées, traduisant comme ailleurs l'émiettement des parcelles agricoles en milieu urbain (Ba, 2007 ; Fanou, 2008 ; Mbaye et Moustier, 2000). Dans toute la zone étudiée, les maraîchers ne sont pas propriétaires des terrains qu'ils exploitent. Dans ce contexte, le paysan sait que la terre pourra être récupérée à tout moment pour la mise en place de l'infrastructure à laquelle elle serait destinée ; ces maraîchers sont ainsi obligés de se déplacer de l'ouest vers l'est. Notre étude montre une dynamique centrifuge des périmètres maraîchers associée à l'urbanisation de la ville de Lomé, qui se caractérise par une concentration et un étalement des constructions sans précédent (Guézéré, 2011). L'insécurité foncière et la pénurie de terres consécutives à l'extension du bâti à vocation industrielle, commerciale ou résidentielle limitent le développement des activités maraîchères (Matthys et al., 2006). Face à cet enjeu, la résolution du problème foncier doit permettre une meilleure organisation de la filière maraîchère pour la ville de Lomé. Les expériences réussies ailleurs dans le domaine comme celle de l'Association pour le Développement Agricole de l'Y Grenoblois (ADAYG), qui a d'ailleurs été à l'origine du réseau Agriville, de Barcelone à Milan (Fleury et Donadieu, 1997), montrent la pertinence de cette pratique qui mériterait un soutien des services publics. Dans ce contexte, la vallée du Zio (au nord de la ville de Lomé) pourrait servir de cadre pour un tel programme maraîcher précédé d'une étude d'impact environnemental afin d'évaluer les risques éventuels. En effet, cette vallée dispose de bons sols et de ressources en eau (Lamouroux, 1969) mais demeure faiblement mise en valeur. Ce programme maraîcher pourrait intégrer et bénéficier à des projets en cours tel que celui de l'élaboration du plan de gestion des zones humides du bassin du Zio. Enfin, il permettrait de freiner l'occupation anarchique des superficies potentiellement agricoles de ce bassin pour des constructions.

\section{Conclusion}

Cette étude avait pour objet d'évaluer la dynamique des superficies maraîchères en réponse à la pression foncière depuis 2002. Elle a permis de montrer que les surfaces maraîchères ont considérablement régressé entre 2002 et 2014 avec comme force motrice principale l'urbanisation. Elle montre également que le marâichage dans la zone étudiée, se pratique sur de petites superficies à statut foncier précaire, contraignant les maraîchers au déplacement sans alternatives. Les résultats de cette étude constituent une information pour la prise de décision en ce qui concerne le développement et l'optimisation de l'agriculture maraîchère dans la ville de Lomé. Dans un contexte d'étalement de cette ville, l'accès à la terre est devenu un frein à l'exercice de cette agriculture malgré le besoin croissant en légumes frais. Il convient de mettre en place une forte action en vue de sa reconnaissance car elle constitue, avec les métiers (emploi, commerce à divers niveaux, approvisionnement en intrants, etc.) qu'elle développe autour d'elle, une opportunité pour le développement d'une économie urbaine.

\section{Références}

Ba A. 2007. Les fonctions reconnues à l'agriculture intra et périurbaines (AIPU) dans le contexte dakarois; caractérisation, analyse et diagnostic de durabilité de cette agriculture en vue de son intégration 
dans le projet urbain de Dakar (Sénégal). Thèse Doct. Sc. Agron. Environnement, AgroParisTech/UCAD/ENSP-Versailles.

Bélières JF, Jamin JY, Seck SM, Tonneau JP, Adamczewski A, Le Gal PY. 2013. Dynamiques foncières, investissements et modèles de production pour l'irrigation en Afrique de l'Ouest : logiques financières contre cohérences sociales ? Cahiers Agricultures 22(1): 61-66.

Bertrand N, Roussier N. 2004. L'agriculture périurbaine, une proximité ville agriculture à construire. Quatrièmes Journées de la Proximité, IDEP-LEST-GREQAM, Groupe de Recherche Dynamique de Proximité, Marseille, 17-18 juin 2004, p. 17.

Billen R, Cornélis B. 2000. Géométrie de la spatiocarte : correction et validation. Bulletin de la Société Géographique de Liège 38(1): 25-42.

Boudemagh BO. 2010. Politiques urbaines, terres agricoles et marché foncier : quel avenir pour l'agriculture périurbaine à Constantine (Algérie) ? Cahiers Agricultures 22(6): 544-551.

Boudjenouia A, Fleury A, Tacherift A. 2008. L'agriculture périurbaine à Sétif (Algérie) : quel avenir face à la croissance urbaine ? Biotechnologie. Agronomie, Société et Environnement 12: 23-30.

Burnod P, Tonneau J-P. 2013. Le foncier : facteur ou marqueur de l'évolution des agricultures ? Cahiers Agricultures 22(1): 4-9.

Cissé G, Odermatt P, Maystre LY, Tanner M. 1999. Utilisation d'un GPS et d'un logiciel de SIG pour évaluer les variations saisonnières des superficies exploitées des sites de maraîchage dans le tissu urbain de Ouagadougou. Sciences et Changements Planétaires Sécheresse 10(2): 123-128.

Dauvergne S. 2012. Les espaces urbains et périurbains à usage agricole dans les villes d'Afrique subsahariennes (Yaoundé et Accra) : une approche de l'intermédiarité en géographie. Thèse doct., UMR Environnement et Sociétés, ENS Lyon.

Delamarche M. 2007. Agriculture urbaine, les villes qui se ruralisent. Marchés tropicaux et méditerranéens : stratégies et investissement en Afrique 32(12): 5-6.

Dieye BM. 2006. Le financement de la production maraîchère : l'exemple de la zone de Potou (Sénégal). BIM 15: 6.

DRSP. 2012. Recensement général de la population et de l'habitat : résultats définitifs. Rapport, Direction Générale de la Statistique et de la Comptabilité Nationale du Togo, Lomé: Togo.

Fanou L. 2008. Rentabilité financière et économique des systèmes de production maraîchers au Sud-Bénin : une application de la matrice d'analyse des politiques. Mém. Ing. Agroéconomiste. Cotonou, Bénin: Université d'Abomey-Calavi. Disponible sur http://www.memoireon line.com/10/09/2750/Rentabilite-financiere-et-economique-des-syste mes-de-production-marachers-au-Sud-Benin-une-a.html.
Fleury A, Donadieu P. 1997. De l'agriculture péri-urbaine à l'agriculture urbaine. Courrier de l'Environnement de l'INRA 31: 45-61.

Guézéré A. 2011. L'obsession d'habiter sa propre maison à Lomé : quel impact sur la dynamique spatiale? Les Cahiers d'Outre-mer 256: $565-590$.

Janssens M. 1998. Lomé, 100 ans de croissance démographique. In : Gayibor N, Marguerat Y, Nyassogbo K, eds. 1897-1997. Lomé, capitale du Togo: Presses de l'Université du Bénin, p. 303-314.

Kanda M, Wala K, Batawila K, Djaneye-Boundjou G, Ahanchédé A, Akpagana K. 2009. L'Agriculture périurbaine à Lomé : diversité spécifique, caractéristiques des agriculteurs, utilisation de pesticides et dynamique spatiale. Cahiers Agricultures 18(4): 356-363.

Kêdowidé CMG, Sedogo MP, Cissé G. 2010. Dynamique spatiotemporelle de l'agriculture urbaine à Ouagadougou: cas du Maraîchage comme une activité montante de stratégie de survie. VertigO 10(2): 1-13.

Kouakou YE, Koné B, Bonfoh B, Kientga SM, N'Go YA, Savane I, et al. 2010. L'étalement urbain au péril des activités agro-pastorales à Abidjan. VertigO 10(2): 1-9.

Lamouroux M. 1969. Carte pédologique du Togo au 1/1 000 000, Notice explicative No. 34. Paris, France: Office de la Recherche Scientifique et Technique Outre-mer (ORSTOM).

Matthys B, Adiko FA, Cissé G, Wyss K, Tschannen AB, Tanner M, et al. 2006. Le réseau social des maraîchers à Abidjan agit sur la perception des préoccupations et des risques sanitaires liés à l'eau. VertigO 3(8): 1-11.

Mbaye A, Moustier P. 2000. Market-oriented urban agricultural production in Dakar. In: Bakker N, et al, eds. Growing cities, growing food: urban agriculture on the policy agenda, a reader on urban agriculture. Allemagne: Feldafing.

Nguyen The N, Ginet C, Giraud A, Bouvet A. 2004. Calcul des surfaces. Atouts et limites des récepteurs GPS « grand public». Revue forestière française 6: 529-536.

Schilter C. 1991. L'agriculture urbaine : une activité créatrice d'emplois, en économie de survie (le cas de Lomé). Cahiers des sciences humaines 27(1-2): 159-168.

Valette E, Chéry JP, Debolini M, Azodjilande J, François M, El Amrani M. 2013. Urbanisation en périphérie de Meknès (Maroc) et devenir des terres agricoles : l'exemple de la coopérative agraire Naïji. Cahiers Agricultures 22: 535-543.

Véron J. 2007. La moitié de la population mondiale vit en ville. Population \& Sociétés 435: 1-4.

Citation de l'article : Kanda M, Badjana HM, Folega F, Akpavi S, Imbernon J, Akpagana K. 2017. Dynamique centrifuge du maraîchage périurbain de Lomé (Togo) en réponse à la pression foncière. Cah. Agric. 26: 15001. 